\title{
Reliability Analysis in Presence of Random Variables and Fuzzy Variables
}

\author{
Cui Lijie, ${ }^{1}$ Lü Zhenzhou, ${ }^{2}$ and Li Guijie ${ }^{2}$ \\ ${ }^{1}$ School of Equipment Management and Safety Engineering, Air Force Engineering University, Xi'an, Shaanxi 710051, China \\ ${ }^{2}$ School of Aeronautics, Northwestern Polytechnical University, Xian, Shaanxi 710072, China \\ Correspondence should be addressed to Cui Lijie; lijie_cui@163.com
}

Received 25 August 2014; Revised 22 January 2015; Accepted 26 January 2015

Academic Editor: Yuri N. Sotskov

Copyright (c) 2015 Cui Lijie et al. This is an open access article distributed under the Creative Commons Attribution License, which permits unrestricted use, distribution, and reproduction in any medium, provided the original work is properly cited.

\begin{abstract}
For mixed uncertainties of random variables and fuzzy variables in engineering, three indices, that is, interval reliability index, mean reliability index, and numerical reliability index, are proposed to measure safety of structure. Comparing to the reliability membership function for measuring the safety in case of mixed uncertainties, the proposed indices are more intuitive and easier to represent the safety degree of the engineering structure, and they are more suitable for the reliability design in the case of the mixed uncertainties. The differences and relations among three proposed indices are investigated, and their applicability is compared. Furthermore, a technique based on the probability density function evolution method is employed to improve the computational efficiency of the proposed indices. At last, a numerical example and two engineering examples are illustrated to demonstrate the feasibility, reasonability, and efficiency of the computational technique of the proposed indices.
\end{abstract}

\section{Introduction}

Uncertainty in engineering can be classified into two different types: aleatory uncertainty and epistemic one. Aleatory uncertainty, referred to as objective, stochastic, is due to inherent variability in the system, and epistemic uncertainty, referred to as subjective, is due to lack of knowledge and information [1-3]. Epistemic uncertainty can be reduced by acquiring knowledge and information in the system, while the aleatory uncertainty cannot, and for this reason it is sometimes called irreducible uncertainty.

Traditionally, the aleatory uncertainty is taken into consideration in reliability-based design, named as random variables, which requires completely statistical information by probability distribution to describe these aleatory uncertainties [4-6]. However, the epistemic uncertainties may not be appropriately represented by the probability distributions, in the case where the fuzzy theory can be used to deal with these epistemic uncertainties, usually named as fuzzy variables, by the membership functions [7-10]. In engineering problems, the random variables and the fuzzy ones exist simultaneously. Therefore, it is important to establish a suitable reliability model to measure the safety of the structure in case of the mixture of random and fuzzy variables. Möller et al. [11] introduced a methodology for estimating the membership function of the safety index by considering fuzzy randomness. They formulated a Fuzzy First Order Reliability Method (FFORM) that simultaneously permits the usage of fuzzy variables and random variable. Using this method, the reliability membership function can be estimated. But the calculation of the failure probability is prone to errors. And stability approaches to the problems with uncertainty are also developed by Lai et al. [12], Matsveichuk et al. [13]. Adduri and Penmetsa $[14,15]$ developed a technique to improve the efficiency of propagating the fuzziness and randomness of the basic variables to the corresponding response variables largely, in which the transformation techniques and the convolution integral by the Fast Fourier Transformation (FFT) are combined to solve the membership function of the response variable. However, the technique depends on the quadratic polynomial without cross-terms (QPWCT); thus it is not suitable for the high nonlinear response function, and the cross-term of random variables and fuzzy variables cannot be included. Furthermore, there is no measure about the safety in Adduri's 
method. In summary, the existed methods dealing with mixture of the fuzzy variables and the random variables are concerned with the membership functions of the reliability index and the response variable. Though the membership functions are relatively complete to describe the fuzzy uncertainties of the reliability and the response, their formulations are not compact and cannot provide an intuitive evaluation of the different designs. Therefore, a series of reliability indices, which can give an intuitive and compact result of the reliability, are proposed in this paper, and a solution of the proposed indices is proposed on the probability density evolution method (PDEM) [16] and it can reduce the computational cost greatly without losing accuracy.

\section{New Reliability Indices with Mixed Variables}

Assuming the performance response function $y$ of the reliability model is given by

$$
\begin{aligned}
y & =g(\mathbf{x})=g\left(\mathbf{x}_{R}, \mathbf{x}_{F}\right) \\
& =g\left(x_{1}, x_{2}, \ldots, x_{n_{R}}, x_{n_{R}+1}, \ldots, x_{n_{R}+n_{F}}\right),
\end{aligned}
$$

where $\mathbf{x}_{R}=\left\{x_{1}, x_{2}, \ldots, x_{n_{R}}\right\}$ are $n_{R}$-dimensional independent random variables, their probability density functions $(\mathrm{PDF})$ are $f_{x_{R_{i}}}\left(x_{R_{i}}\right)\left(R_{i}=1,2, \ldots, n_{R}\right)$, and where $\mathbf{x}_{F}=$ $\left\{x_{n_{R}+1}, \ldots, x_{n_{R}+n_{F}}\right\}$ are $n_{F}$-dimensional fuzzy variables, their membership functions are $\mu_{x_{F_{i}}}\left(x_{F_{i}}\right)\left(F_{i}=n_{R}+1, \ldots, n_{R}+n_{F}\right)$.

According to the reliability theory, the reliability $P_{r}$ and the failure probability $P_{f}$ of the performance response function $g\left(\mathbf{x}_{R}, \mathbf{x}_{F}\right)$ can be defined by

$$
\begin{gathered}
P_{r}=P\left\{g\left(\mathbf{x}_{R}, \mathbf{x}_{F}\right)>0\right\}, \\
P_{f}=P\left\{g\left(\mathbf{x}_{R}, \mathbf{x}_{F}\right) \leq 0\right\}=1-P_{r} .
\end{gathered}
$$

Since the performance function $g\left(\mathbf{x}_{R}, \mathbf{x}_{F}\right)$ involves $\mathbf{x}_{R}$ and $\mathbf{x}_{F}, P_{r}$ and $P_{f}$ possess fuzziness resulted from $\mathbf{x}_{F}$ through $g\left(\mathbf{x}_{R}, \mathbf{x}_{F}\right)$.

The fuzzy variables can be evaluated by $\lambda$-cuts [7], shown in Figure 1, where $\lambda$ is a given membership level. Different $\lambda$-cuts correspond to different confidence bounds marked by confidence $\lambda$. Once the basic variables are defined by the fuzzy membership functions, the $\lambda$-cuts of the reliability $P_{r}$ and the failure probability $P_{f}$ at various membership level $\lambda$ can be obtained according to the relations shown in (2). Figure 2 shows the transformation from the membership function of the basic fuzzy variables of $\mathbf{x}_{F}$ to that of the failure probability $P_{f}$.

Denote $\lambda$-cut $\mathbf{x}_{F}(\lambda)$ of the fuzzy variables $\mathbf{x}_{F}$ as $\mathbf{x}_{F}(\lambda)=$ $\left[\underline{\mathbf{x}}_{F}(\lambda), \overline{\mathbf{x}}_{F}(\lambda)\right]$, where $\underline{\mathbf{x}}_{F}(\lambda)$ and $\overline{\mathbf{x}}_{F}(\lambda)$ are the lower and upper bounds of the $\lambda$-cut of $\mathbf{x}_{F}$. Then combining optimization or the traversal method with the traditional random reliability analysis methods, the $\lambda$-cut $P_{r}(\lambda)=\left[\underline{P}_{r}(\lambda), \bar{P}_{r}(\lambda)\right]$ of $P_{r}$ and the $\lambda$-cut $P_{f}(\lambda)=\left[\underline{P}_{f}(\lambda), \bar{P}_{f}(\lambda)\right]$ of $P_{f}$ can be obtained, where $\underline{P}_{r}(\lambda)$ and $\underline{P}_{f}(\lambda)$ are the lower bounds of the $\lambda$-cut of $P_{r}$

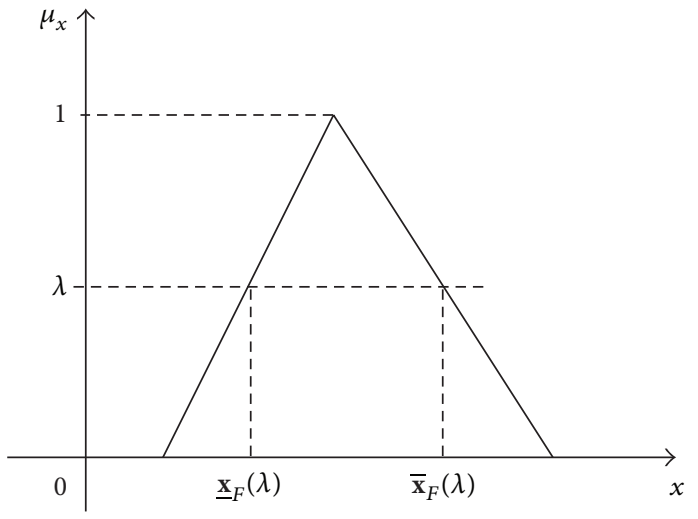

FIgURE 1: The membership function showing an $\lambda$-cut.

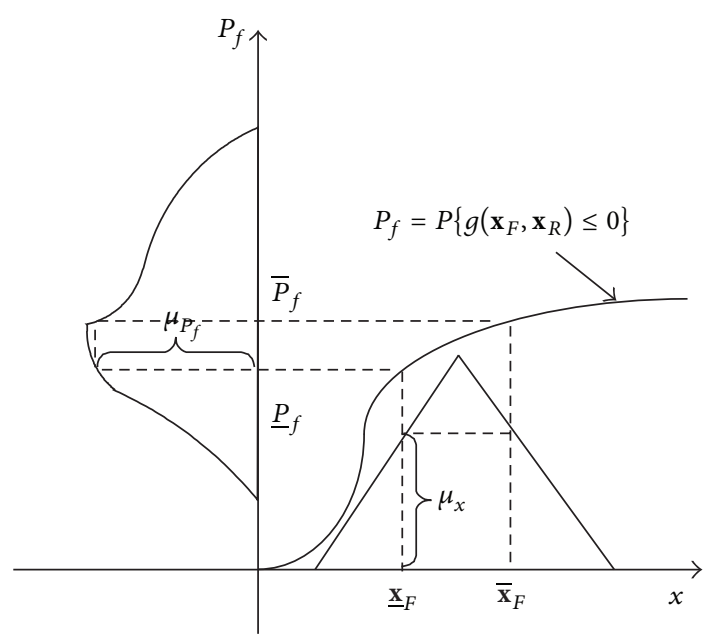

FIgURE 2: Transformation technique of membership function.

and that of $P_{f}$, respectively; $\bar{P}_{r}(\lambda)$ and $\bar{P}_{f}(\lambda)$ are the corresponding upper bounds. The relations between $P_{r}(\lambda), P_{f}(\lambda)$, and $\mathbf{x}_{F}(\lambda)$ are listed, respectively, as follows:

$$
\begin{gathered}
\bar{P}_{r}(\lambda)=\max \left(P\left\{g\left(\mathbf{x}_{R}, \mathbf{x}_{F}\right)>0 \mid \mathbf{x}_{F} \in \mathbf{x}_{F}(\lambda)\right\}\right), \\
\underline{P}_{r}(\lambda)=\min \left(P\left\{g\left(\mathbf{x}_{R}, \mathbf{x}_{F}\right)>0 \mid \mathbf{x}_{F} \in \mathbf{x}_{F}(\lambda)\right\}\right), \\
\bar{P}_{f}(\lambda)=\max \left(P\left\{g\left(\mathbf{x}_{R}, \mathbf{x}_{F}\right) \leq 0 \mid \mathbf{x}_{F} \in \mathbf{x}_{F}(\lambda)\right\}\right), \\
\underline{P}_{f}(\lambda)=\min \left(P\left\{g\left(\mathbf{x}_{R}, \mathbf{x}_{F}\right) \leq 0 \mid \mathbf{x}_{F} \in \mathbf{x}_{F}(\lambda)\right\}\right) .
\end{gathered}
$$

Since equalities $P_{r}\left(\mathbf{x}_{F}^{*}\right)=P\left\{g\left(\mathbf{x}_{R}, \mathbf{x}_{F}^{*}\right)>0\right\}, P_{f}\left(\mathbf{x}_{F}^{*}\right)=$ $P\left\{g\left(\mathbf{x}_{R}, \mathbf{x}_{F}^{*}\right) \leq 0\right\}$, and $P_{r}\left(\mathbf{x}_{F}^{*}\right)+P_{f}\left(\mathbf{x}_{F}^{*}\right)=1$ hold when $\mathbf{x}_{F}=\mathbf{x}_{F}^{*}$, the following properties about the bounds of the $\lambda$-cut of $P_{r}$ and those of the $\lambda$-cut of $P_{f}$ hold:

$$
\begin{aligned}
& \bar{P}_{r}(\lambda)+\underline{P}_{f}(\lambda)=1, \\
& \underline{P}_{r}(\lambda)+\bar{P}_{f}(\lambda)=1 .
\end{aligned}
$$

By taking the value of the membership level $\lambda$ from 0 to 1, the membership functions $\mu_{P_{r}}\left(P_{r}\right)$ and $\mu_{P_{f}}\left(P_{f}\right)$ of $P_{r}$ and 
$P_{f}$ can be obtained by the decomposition theorem in the fuzzy theory [17]. Obviously, the membership functions $\mu_{P_{r}}\left(P_{r}\right)$ and $\mu_{P_{f}}\left(P_{f}\right)$ can completely reflect the fuzziness of the reliability $P_{r}$ and the failure probability $P_{f}$ in the presence of the random variables and the fuzzy variables, but the drawback is that the membership function cannot intuitively and compactly reflect the degree of safety with mixed uncertainties; it is inconvenient for comparing the safety degrees of the different products and the reliability based design optimization. Generally, it is not easy for the implicit performance function to solve (3), either. According to these drawbacks of the membership function of the reliability or the failure probability with mixed variables, the following new indices are proposed on the basis of the membership function characteristic.

2.1. Reliability Index I: Interval Reliability Index. The interval reliability index is defined by the upper bounds $\bar{P}_{r}^{(\mathrm{I})}$ and $\bar{P}_{f}^{(\mathrm{I})}$ and the lower bounds $\underline{P}_{r}^{(\mathrm{I})}$ and $\underline{P}_{f}^{(\mathrm{I})}$ computed by

$$
\begin{aligned}
& \underline{P}_{r}^{(\mathrm{I})}=\int_{0}^{1} \underline{P}_{r}(\lambda) d \lambda, \\
& \bar{P}_{r}^{(\mathrm{I})}=\int_{0}^{1} \bar{P}_{r}(\lambda) d \lambda, \\
& \underline{P}_{f}^{(\mathrm{I})}=\int_{0}^{1} \underline{P}_{f}(\lambda) d \lambda, \\
& \bar{P}_{f}^{(\mathrm{I})}=\int_{0}^{1} \bar{P}_{f}(\lambda) d \lambda,
\end{aligned}
$$

where indices $\bar{P}_{r}^{(\mathrm{I})}, \bar{P}_{f}^{(\mathrm{I})}$ and $\underline{P}_{r}^{(\mathrm{I})}, \underline{P}_{f}^{(\mathrm{I})}$ represent the expectations of the maximum and the minimum of reliability $P_{r}$ and the failure probability $P_{f}$, respectively, when the membership level $\lambda$ is assumed to obey the uniform distribution over interval $[0,1]$. Using the properties shown in (4), the following equations hold:

$$
\begin{aligned}
& \bar{P}_{r}^{(\mathrm{I})}+\underline{P}_{f}^{(\mathrm{I})}=1, \\
& \underline{P}_{r}^{(\mathrm{I})}+\bar{P}_{f}^{(\mathrm{I})}=1 .
\end{aligned}
$$

2.2. Reliability Index II: Mean Reliability Index. Taking the mean values of $\left[\underline{P}_{f}^{(\mathrm{I})}, \bar{P}_{f}^{(\mathrm{I})}\right]$ and $\left[\underline{P}_{r}^{(\mathrm{I})}, \bar{P}_{r}^{(\mathrm{I})}\right]$ as the second failure probability index $P_{f}^{(\mathrm{II})}$ and reliability index $P_{r}^{(\mathrm{II})}$, respectively, we can obtain the more compact reliability indices defined in (7), which are named as mean reliability indices:

$$
\begin{aligned}
& P_{r}^{(\mathrm{II})}=\frac{1}{2}\left[\underline{P}_{r}^{(\mathrm{I})}+\bar{P}_{r}^{(\mathrm{I})}\right]=\frac{1}{2} \int_{0}^{1}\left[\underline{P}_{r}(\lambda)+\bar{P}_{r}(\lambda)\right] d \lambda, \\
& P_{f}^{(\mathrm{II})}=\frac{1}{2}\left[\underline{P}_{f}^{(\mathrm{I})}+\bar{P}_{f}^{(\mathrm{I})}\right]=\frac{1}{2} \int_{0}^{1}\left[\underline{P}_{f}(\lambda)+\bar{P}_{f}(\lambda)\right] d \lambda .
\end{aligned}
$$

Similarly, the following relation exists between $P_{r}^{(\mathrm{II})}$ and $P_{f}^{(\mathrm{II})}$ :

$$
P_{r}^{(\mathrm{II})}+P_{f}^{(\mathrm{II})}=1
$$

2.3. Reliability Index III: Numerical Reliability Index. The above two new reliability indices require the optimization methods or the transformation method to solve the upper and the lower bounds of the reliability and the failure probability at each membership level. However, it is difficult to compute them for the implicit performance function $g\left(\mathbf{x}_{R}, \mathbf{x}_{F}\right)$. In fact, when $\mathbf{x}_{F}(\lambda) \in\left[\underline{\mathbf{x}}_{F}(\lambda), \overline{\mathbf{x}}_{F}(\lambda)\right]$ for the given membership level $\lambda$, we can assume that $\mathbf{x}_{F}(\lambda)$ is the uniform distribution vector in $\left[\underline{\mathbf{x}}_{F}(\lambda), \overline{\mathbf{x}}_{F}(\lambda)\right]$. After the fuzzy basic variables are assumed as the uniform variables at the given $\lambda$, the traditional reliability analysis method can be used to compute the reliability $P_{r}(\lambda)$ and the failure probability $P_{f}(\lambda)$. Taking values of $\lambda$ from 0 to 1 , the numerical reliability indices, that is, a new reliability index $P_{r}^{(\mathrm{III})}$ and a new failure probability index $P_{f}^{(\mathrm{III})}$, are defined by

$$
\begin{aligned}
& P_{r}^{(\mathrm{III})=} \int_{0}^{1} P\left\{g\left(\mathbf{x}_{R}, \mathbf{x}_{F}(\lambda)\right)>0\right\} d \lambda \\
&= \int_{0}^{1}\left\{\int_{g\left(\mathbf{x}_{R}, \mathbf{x}_{F}(\lambda)\right)>0} f_{\mathbf{x}_{R}}\left(\mathbf{x}_{R}\right)\right. \\
&\left.\cdot f_{\mathbf{x}_{F}(\lambda)}\left(\mathbf{x}_{F}(\lambda)\right) d \mathbf{x}_{R} d \mathbf{x}_{F}(\lambda)\right\} d \lambda, \\
& P_{f}^{(\mathrm{III})}=\int_{0}^{1} P\left\{g\left(\mathbf{x}_{R}, \mathbf{x}_{F}(\lambda)\right) \leq 0\right\} d \lambda \\
&=\int_{0}^{1}\left\{\int_{g\left(\mathbf{x}_{R}, \mathbf{x}_{F}(\lambda)\right) \leq 0} f_{\mathbf{x}_{R}}\left(\mathbf{x}_{R}\right)\right. \\
&\left.\cdot f_{\mathbf{x}_{F}(\lambda)}\left(\mathbf{x}_{F}(\lambda)\right) d \mathbf{x}_{R} d \mathbf{x}_{F}(\lambda)\right\} d \lambda,
\end{aligned}
$$

where $f_{\mathbf{x}_{F}(\lambda)}\left(\mathbf{x}_{F}(\lambda)\right)$ is the joint PDF of the assumed uniformly distributed $\mathbf{x}_{F}(\lambda)$ and $f_{\mathbf{x}_{R}}\left(\mathbf{x}_{R}\right)$ is the joint PDF of the random basic variables $\mathbf{x}_{R}$.

Obviously, the following property holds by the definitions of the numerical reliability indices:

$$
P_{r}^{(\mathrm{III})}+P_{f}^{(\mathrm{III})}=1 .
$$

2.4. Comparison of New Indices. Comparing with the membership functions of the reliability and the failure probability, the above three indices can provide more intuitive and compact results to represent the safety degree in the case of the mixed variables. They take the characters of the reliability membership function from different perspectives; thus these proposed indices can be named as the characteristic indices of the reliability membership function. The interval reliability 
index uses an interval to represent the safety degree of the structure, which reflects the expectation of the extreme value of the reliability and the failure probability as the membership level obeying the uniform distribution in $[0,1]$. The mean reliability index evaluates the reliability from the perspective of the mean of the interval reliability index, while the numerical index directly transforms the fuzzy variables in the $\lambda$-cuts into the uniformly distributed random variables and then uses the traditional random reliability analysis methods and the fuzzy theory to obtain the numerical results. The numerical reliability index is equivalent to the expectations of the reliability, in which the membership level $\lambda$ distributes in $[0,1]$ uniformly and the fuzzy basic variables distribute in the $\lambda$-cuts uniformly.

Analyzing their physical properties, we can find some relations among the proposed three indices. The mean reliability index is always the mean value of the interval one. The definition of the numerical index is explicitly described by the expectation of supposing the fuzzy basic variables as uniformly random variables, and it can be conveniently solved by the traditional random reliability methods. When the reliability membership function is symmetrical or there exists linear relation between the reliability and the fuzzy basic variables, the mean reliability index is equal to the numerical one. But in most engineering problems, the reliability membership functions are not symmetrical and there exists no complete linear relation between the reliability and the fuzzy basic variables; thus the mean reliability indices are not equal to the numerical indices in this case.

In order to solve the interval reliability index and the mean one, the extreme value of the reliability must be obtained at each membership level $\lambda$ by the optimum method or the traversal methods; their computational costs are unacceptable for some implicit performance functions or complex models. But the solution of the numerical reliability index does not require the optimum or the traversal process. By use of the traditional reliability methods, the solution of the numerical reliability index is predigested largely. What is more, the proposed numerical indices provide convenient approaches to reliability optimization design. Firstly, in the optimization design, it is difficult to optimize the membership function but it is feasible to optimize the characteristic indices of the reliability membership function. Secondly, the sensitivity analysis of the characteristic indices of the reliability membership function could give the proper optimization directions, while it is impracticable to the membership function index.

\section{The PDEM-Based Solution of New Reliability Indices}

It is inevitable to compute the reliability or the failure probability in solving new reliability indices. The MCM and its adaptive methods are general methods to obtain the reliability or the failure probability of structures with random variables [18-20]. To obtain the interval reliability index and the mean reliability index with mixed variables, when the membership level is $\lambda$, the fuzzy variables $\mathbf{x}_{F}$ take fixed value $\mathbf{x}_{F}^{*}$ within the $\lambda$-cuts $\left[\underline{\mathbf{x}}_{F}(\lambda), \overline{\mathbf{x}}_{F}(\lambda)\right]$, and the reliability $P_{r}^{*}(\lambda)=$ $P\left\{g\left(\mathbf{x}_{R}, \mathbf{x}_{F}^{*}\right)>0\right\}$ or the failure probability $P_{f}^{*}(\lambda)=P\left\{g\left(\mathbf{x}_{R}\right.\right.$, $\left.\left.\mathbf{x}_{F}^{*}\right) \leq 0\right\}$ can be got by the traditional reliability methods; then the maximum and the minimum of the reliability or the failure probability as $\mathbf{x}_{F} \in\left[\underline{\mathbf{x}}_{F}(\lambda), \overline{\mathbf{x}}_{F}(\lambda)\right]$, that is, the $\lambda$-cuts of the reliability or the failure probability, $P_{r}(\lambda)$ and $P_{f}(\lambda)$, can be obtained by using the optimization method or the traversal method. Furthermore, the interval reliability index $\left[\underline{P}_{r}^{(\mathrm{I})}, \bar{P}_{r}^{(\mathrm{I})}\right]$ and $\left[\underline{P}_{f}^{(\mathrm{I})}, \bar{P}_{f}^{(\mathrm{I})}\right]$ can be obtained by (5); the mean reliability index $P_{r}^{(\mathrm{II})}$ and $P_{f}^{(\mathrm{II})}$ can be obtained by (7). To obtain the numerical reliability index, firstly, the fuzzy variables $\mathbf{x}_{F}$ are transformed into the uniformly distributed random variables in $\left[\underline{\mathbf{x}}_{F}(\lambda), \overline{\mathbf{x}}_{F}(\lambda)\right]$; namely, the performance response function with mixed variables is transformed into the performance response function with random variables; the reliability $P_{r}(\lambda)$ and the failure probability $P_{f}(\lambda)$ can be computed by the random reliability analysis methods; then the numerical reliability index $P_{r}^{(\mathrm{III})}$ and $P_{f}^{(\mathrm{III})}$ with mixed variables can be obtained by combining with the decomposition theorem and (9).

However, the MCM and its adaptive methods involve tremendous computational cost, which is impossible in most engineering problems. Therefore, there is a clear requirement to develop probability integration schemes that are applicable for these problems.

Recently developed PDEM provides a high efficient method to solve the PDF $f_{Y}(y)$ of the performance response function $Y=g(\mathbf{x})$. If the PDF $f_{Y}(y)$ can be obtained, the reliability $P_{r}$ and the failure probability $P_{f}$ can be computed conveniently by

$$
\begin{aligned}
& P_{r}=P(g(\mathbf{x})>0)=\int_{0}^{+\infty} f_{Y}(y) d y, \\
& P_{f}=P(g(\mathbf{x}) \leq 0)=\int_{-\infty}^{0} f_{Y}(y) d y .
\end{aligned}
$$

Due to the uncertainty of the performance function $g(\mathbf{x})$ completely originating from uncertain input basic variable, a virtual stochastic process with $\tau$ as the virtual time can be constructed as

$$
Y(\tau)=\varphi(g(\mathbf{x}), \tau)=\phi(\mathbf{x}, \tau)
$$

and the virtual stochastic process satisfies the conditions $\left.Y(\tau)\right|_{\tau=0}=0$ and $\left.Y(\tau)\right|_{\tau=\tau_{c}}=g(\mathbf{x})$, where $\tau_{c}$ is the end of the virtual time.

Denote $f_{Y \mathbf{X}}(y, \mathbf{x}, \tau)$ as the joint PDF of variables $Y(\tau)$ and $\mathbf{x}$; then according to the probability conservation law [21], the generalized probability density evolution equation can be constructed as

$$
\frac{\partial f_{Y \mathbf{X}}(y, \mathbf{x}, \tau)}{\partial \tau}+\dot{\phi}(\mathbf{x}, \tau) \frac{\partial f_{Y \mathbf{X}}(y, \mathbf{x}, \tau)}{\partial y}=0
$$

and the initial condition is

$$
\left.f_{Y \mathbf{X}}(y, \mathbf{x}, \tau)\right|_{\tau=0}=\delta(y) f_{\mathbf{X}}(\mathbf{x}),
$$

where $\dot{\phi}(\mathbf{x}, \tau)=\partial \phi(\mathbf{x}, \tau) / \partial \tau$ and $\delta(\cdot)$ is the Dirac function. 
$f_{Y \mathbf{X}}(y, \mathbf{x}, \tau)$ can be obtained by solving the above partial derivative equation, and $f_{Y}(y, \tau)$ also can be obtained by

$$
f_{Y}(y, \tau)=\int_{\Omega_{\mathbf{X}}} f_{Y \mathbf{X}}(y, \mathbf{x}, \tau) d \mathbf{x} .
$$

Since $Y\left(\tau_{c}\right)=g(\mathbf{x})=Y$, the PDF $f_{Y}(y)$ of the performance response function $Y=g(\mathbf{x})$ can be got by

$$
f_{Y}(y)=\left.f_{Y}(y, \tau)\right|_{\tau=\tau_{c}} .
$$

In computational fluid dynamics [22], there are many algorithms to solve the hyperbolic conservational partial derivative equation as (13). Among the strategies of selecting representative points of $\mathbf{x}$, the number theoretical method can improve computational efficiency by reducing the dimension of the input basic variables [23, 24]. In the finite difference method for solving the equation, the difference scheme with TVD nature or combined difference scheme can obtain more precise result and reduce oscillatory and dissipation in process of difference [16].

\section{Examples}

Three examples including a numerical example and two engineering examples are used to demonstrate the feasibility precision of the proposed indices and the efficiency of the presented PDEM-based solution. For comparison, results of three indices calculated by the Monte Carlo method (MCM) are referred to as exact ones.

Example 1 (numerical example). The performance response function [6] is given as

$$
g(\mathbf{x})=x_{1}^{2}+5 x_{1}+2 x_{2}^{2}+7 x_{2}+x_{3}^{2}-8 x_{3}+x_{4}^{2}-10 x_{4}-200,
$$

where $x_{1}$ and $x_{2}$ are assumed to be normally distributed with a mean of 10.0 and a standard deviation of 2.0. The variables $x_{3}$ and $x_{4}$ are assumed to be fuzzy variables, and their membership functions are given by

$$
\mu_{x_{i}}\left(x_{i}\right)=\left\{\begin{array}{ll}
\frac{\left(x_{i}-5\right)}{5} & 5 \leq x_{i} \leq 10 \\
\frac{\left(15-x_{i}\right)}{5} & 10 \leq x_{i} \leq 15
\end{array} \quad(i=3,4) .\right.
$$

Figure 3 shows the membership functions of the failure probability $P_{f}$ estimated using the proposed PDEM-based methodology and the Monte Carlo method (MCM), and Table 1 displays three proposed reliability indices computed by two methods, respectively. The results computed by two methods are matched approximately, which testify that the PDEM-based solution can improve the computational efficiency largely without losing accuracy. For example, each failure probability needs to compute the performance function at least $10^{6}$ runs by the MCM, while that only needs 192 runs by the PDEM-based solution. It also can be found that the mean reliability index $P_{f}^{(\mathrm{II})}$ is the mean value of the interval reliability index $P_{f}^{(\mathrm{I})}$; the numerical reliability index $P_{f}^{(\mathrm{III})}$ reflects

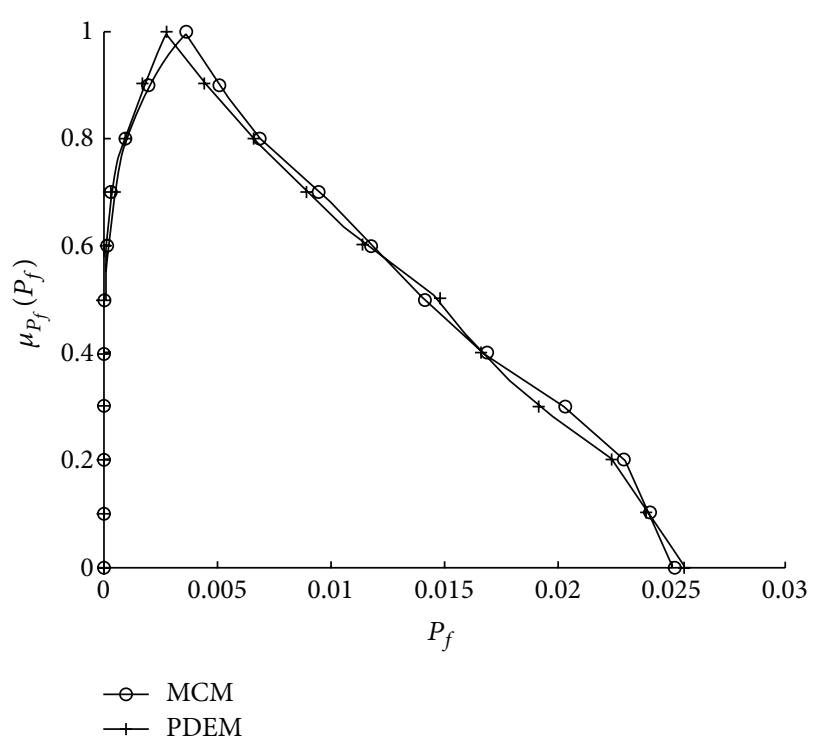

FIgURE 3: The membership function of $P_{f}$ of Example 1.

TABle 1: The reliability indices of Example 1.

\begin{tabular}{lccc}
\hline & $P_{f}^{(\mathrm{I})}$ & $P_{f}^{(\mathrm{II})}$ & $P_{f}^{(\mathrm{III})}$ \\
\hline PDEM & {$[0.000559,0.014275]$} & 0.007417 & 0.003549 \\
MCM & {$[0.000636,0.014555]$} & 0.007595 & 0.003870 \\
\hline
\end{tabular}

the safety degree in case of the mixture of random variables and fuzzy variables from the perspective of supposing the fuzzy variables $x_{3}$ and $x_{4}$ obeying the uniform distribution in their $\lambda$-cuts, and it is more approximate to the left side of the above membership function in Figure 3.

Example 2 (roof truss structure). A roof truss [25] is shown in Figure 4, the top boom and the compression bars are reinforced by concrete, and the bottom boom and the tension bars are steel. The uniform load $q$ (units: $\mathrm{N} / \mathrm{m}$ ) is applied on the roof truss, it is assumed as a fuzzy set due to lack of information and its membership function is constructed as

$$
\mu_{q}(q)= \begin{cases}\frac{(q-19000)}{1000} & 19000 \leq q \leq 20000 \\ \frac{(21000-q)}{1000} & 20000 \leq q \leq 21000 .\end{cases}
$$

Considering the safety and applicability, the perpendicular deflection $\Delta_{C}$ of the peak of structure node $C$ not exceeding $3 \mathrm{~cm}$ is taken as the constraint condition, where $\Delta_{C}$ is the function of the basic variables, and $\Delta_{C}=$ $\left(q l^{2} / 2\right)\left(3.81 / A_{C} E_{C}+1.13 / A_{S} E_{S}\right) ; A_{C}, A_{S}, E_{C}, E_{S}$, and $l$ are sectional area, elastic modulus, and length of the concrete and steel bars, respectively; the distribution parameters of the independent normally random variables are given in Table 2.

Figure 5 displays the membership functions of the failure probability and Table 3 lists results of the proposed indices of the roof truss structure by two methods, respectively. 


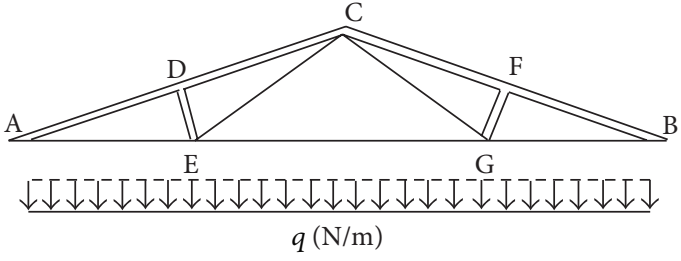

(a)

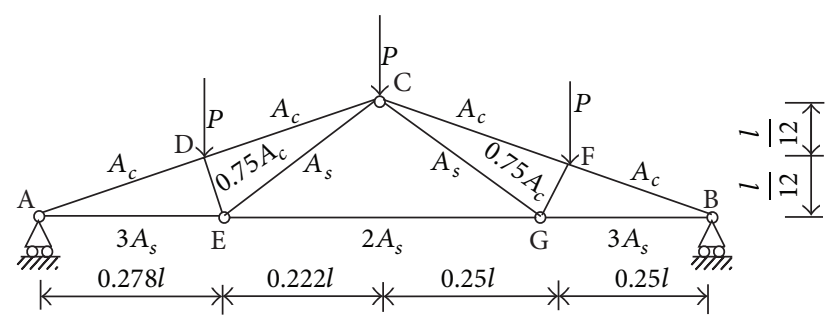

(b)

FIgURE 4: The schematic diagram of a roof truss.

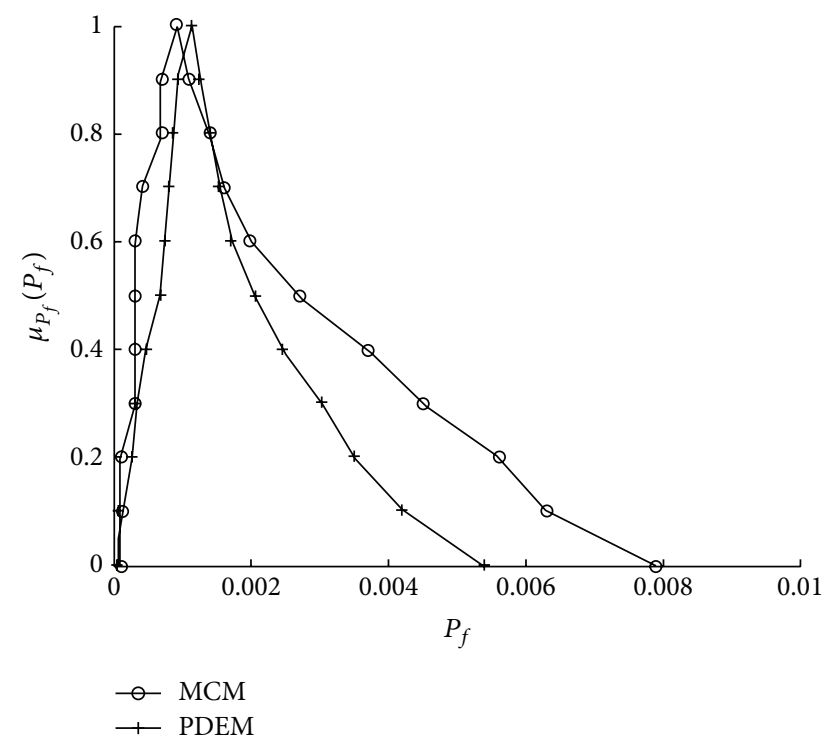

FIGURE 5: The membership function of $P_{f}$ of the roof truss structure.

TABLE 2: The distributional parameters of the basic random variables of roof truss.

\begin{tabular}{lccccc}
\hline $\begin{array}{l}\text { Random } \\
\text { variable }\end{array}$ & $l(\mathrm{~m})$ & $A_{S}\left(\mathrm{~m}^{2}\right)$ & $A_{C}\left(\mathrm{~m}^{2}\right)$ & $E_{S}\left(\mathrm{~N} / \mathrm{m}^{2}\right)$ & $E_{C}\left(\mathrm{~N} / \mathrm{m}^{2}\right)$ \\
\hline Mean $\mu_{x}$ & 12 & $9.82 \times 10^{-4}$ & 0.04 & $1 \times 10^{11}$ & $2 \times 10^{10}$ \\
\hline $\begin{array}{l}\text { Coefficient of } \\
\text { variance } \operatorname{cov}_{x}\end{array}$ & 0.01 & 0.06 & 0.12 & 0.06 & 0.06 \\
\hline
\end{tabular}

TABLE 3: The reliability indices of roof truss structure.

\begin{tabular}{lccc}
\hline & $P_{f}^{(\mathrm{I})}$ & $P_{f}^{(\mathrm{II})}$ & $P_{f}^{(\mathrm{III})}$ \\
\hline PDEM & {$[0.000583,0.002516]$} & 0.001549 & 0.001270 \\
MCM & {$[0.000382,0.003427]$} & 0.001905 & 0.001220 \\
\hline
\end{tabular}

Example 3 (the shaper mechanism). The shaper mechanism [26] (in Figure 6) is a leader-slider mechanism; the mechanism makes the linkage $l_{2}$ swing left and right around the point $O_{2}$ by turning the winch $l_{1}$ at angular velocity and taking the slider A moving along the straight line, which finishes two work processes including a work stroke and a void stroke. In the mechanism, $l_{1}, l_{2}, l_{3}$ are modeled as normally distributed random variables with mean values of $0.1 \mathrm{~m}, 0.75 \mathrm{~m}, 0.738 \mathrm{~m}$

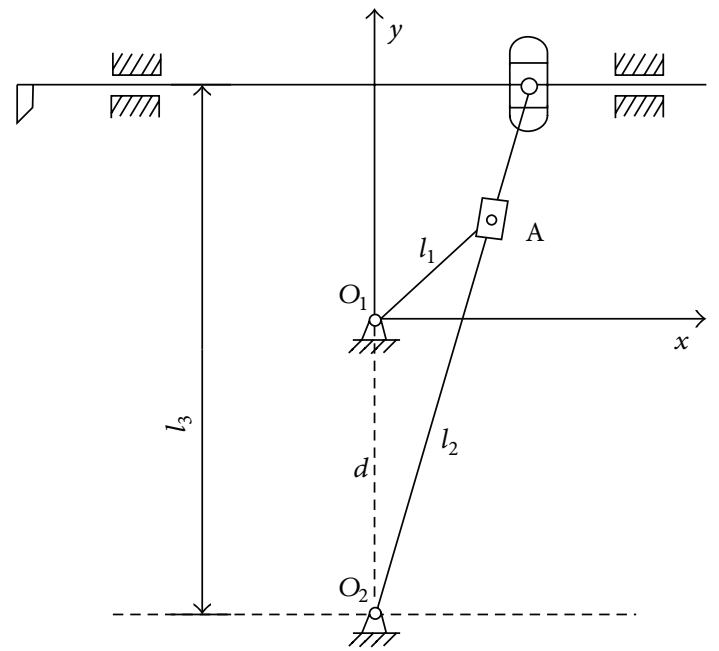

Figure 6: The shaper mechanism scheme.

and the coefficients of variation are assumed to be 0.001 for all of these random variables. The distance $d$ (units: $\mathrm{m}$ ) between points $O_{1}$ and $O_{2}$ is assumed as a fuzzy variable due to lack of knowledge; its fuzzy membership function is

$$
\mu_{d}(d)= \begin{cases}\frac{(d-0.3988)}{0.0012} & 0.3988 \leq d \leq 0.4 \\ \frac{(0.4012-d)}{0.0012} & 0.4 \leq d \leq 0.4012 .\end{cases}
$$

To keep high process precision in its work stroke namely that the error of the shave's velocity must not exceed the allowable value. Denote $[V]$ as the allowable value, $\mathbf{x}$ as the basic input variables, $v(\mathbf{x}, t)$ as practical velocity response, and $v^{*}(\mathbf{x}, t)$ as the ideal velocity response within its work stroke. Construct the performance response function as

$$
Y(\mathbf{x}, t)=g(\mathbf{x}, t)=[V]-\left|v(\mathbf{x}, t)-v^{*}(\mathbf{x}, t)\right|, \quad t \in\left[t_{1}, t_{2}\right] .
$$

Obviously, since inputs $\mathbf{x}$ are input variables with uncertainty, the output response $Y(\mathbf{x}, t)$ must be an uncertain process; namely, the performance function is an uncertain performance process, which also can be called the performance response uncertain process. Generally, the solution of $g(\mathbf{x}, t)$ needs to solve infinite uncertain function, which is 
impossible in fact. But if at any point $t_{i}$ of the process, the performance function $g\left(\mathbf{x}, t_{i}\right)>0$, the mechanism's motion must be safe [27]. That is to say, in whole work stroke, if the minimum of the performance response uncertain process $\min _{t \in\left[t_{1}, t_{2}\right]}(g(\mathbf{x}, t))>0$, the mechanism must be safe and reliable in its work stroke. Therefore, the reliability of mechanism kinematic accuracy $P_{r}$ is computed as

$$
P_{r}=P\left(\bigcap_{t_{i} \in\left[t_{1}, t_{2}\right]} g\left(\mathbf{x}, t_{i}\right)>0\right)=P\left(\min _{t \in\left[t_{1}, t_{2}\right]}(g(\mathbf{x}, t))>0\right) .
$$

Similarly, the failure probability of mechanism kinematic accuracy $P_{f}$ is computed as

$$
\begin{aligned}
P_{f} & =P\left(\bigcup_{t_{i} \in\left[t_{1}, t_{2}\right]} g\left(\mathbf{x}, t_{i}\right) \leq 0\right)=P\left(\min _{t \in\left[t_{1}, t_{2}\right]}(g(\mathbf{x}, t)) \leq 0\right) \\
& =1-R .
\end{aligned}
$$

As $t \in\left[t_{1}, t_{2}\right]$, denote the minimum of $g(\mathbf{x}, t)$ as $z_{\min , T}$; namely,

$$
z_{\min , T}=\min _{t \in\left[t_{1}, t_{2}\right]}(g(\mathbf{x}, t))=\min _{t \in\left[t_{1}, t_{2}\right]}\left([V]-\left|v(\mathbf{x}, t)-v^{*}(\mathbf{x}, t)\right|\right) .
$$

If PDF of the minimum of the performance stochastic process $g(\mathbf{x}, t)$, that is, $f_{z_{\min , T}}(z)$, can be solved, the reliability $P_{r}$ and the failure probability $P_{f}$ of the mechanism kinematic accuracy can be computed by

$$
\begin{aligned}
P_{r} & =P\left(\bigcap_{t_{i} \in\left[t_{1}, t_{2}\right]} g\left(\mathbf{x}, t_{i}\right)>0\right)=P\left(\min _{t \in\left[t_{1}, t_{2}\right]}(g(\mathbf{x}, t))>0\right) \\
& =\int_{0}^{+\infty} f_{z_{\min , T}}(z) d z, \\
P_{f} & =P\left(\bigcup_{t_{i} \in\left[t_{1}, t_{2}\right]} g\left(\mathbf{x}, t_{i}\right) \leq 0\right)=P\left(\min _{t \in\left[t_{1}, t_{2}\right]}(g(\mathbf{x}, t)) \leq 0\right) \\
& =\int_{-\infty}^{0} f_{z_{\min , T}}(z) d z .
\end{aligned}
$$

According to the requirement of the shaper mechanism, the allowable error of the velocity can be assumed as $[V]=$ $0.0016 \mathrm{~m} / \mathrm{s}$. The membership functions of the failure probability and the reliability indices proposed in the paper can be computed by the PDEM-based solution and the MCM, respectively, and their results are shown in Figure 7 and Table 4.

Examples 1 and 3 applied the proposed reliability indices and presented methods into two engineering reliability models with mixture of random variables and fuzzy variables. Three indices including the interval reliability index, the mean reliability index, and the numerical reliability index are

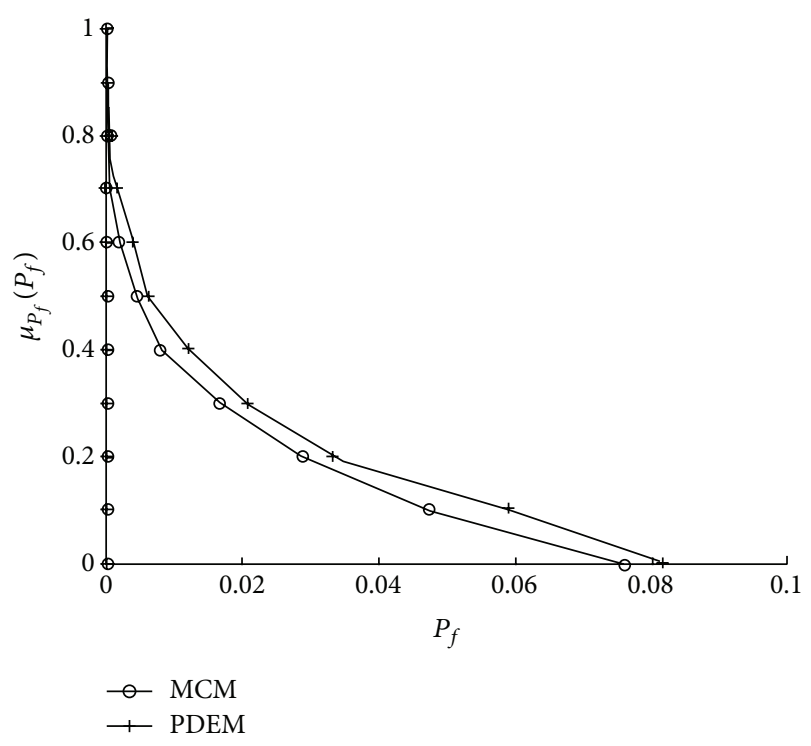

Figure 7: The membership function of $P_{f}$ of Example 2.

TABle 4: The reliability indices of Example 1.

\begin{tabular}{lccc}
\hline & $P_{f}^{(\mathrm{I})}$ & $P_{f}^{(\mathrm{II})}$ & $P_{f}^{(\mathrm{III})}$ \\
\hline PDEM & {$[0,0.019983]$} & 0.010042 & 0.003638 \\
MCM & {$[0,0.016791]$} & 0.008395 & 0.003486 \\
\hline
\end{tabular}

listed in Tables 3 and 4, and membership functions of two examples are displayed in Figures 5 and 7. From that some conclusions can be drawn. Firstly, the proposed indices can reflect the safe degree of the structure and mechanism correctly. Among these reliability indices, the interval reliability index reflects the safe degree in case of the structure with mixed variables from the perspective of the expectation of maximum and the minimum of the failure probability and the mean reliability index provides the mean value of the interval reliability index, while the numerical reliability index reflects the safety degree of the structure/mechanism as supposing the fuzzy basic variables as the uniformly distributed random variables in their $\lambda$-cuts. Secondly, results computed by the PDEM-based method are approximate to that computed by the MCM but which have a large advancement in computational efficiency, which provide an efficient and precise solution to solve the reliability of structure or mechanism in case of mixed variables. Of course, the more complex the model is, the larger the computational error of the PDEMbased method is, which can be solved by enhancing their computational costs and net density in difference arithmetic. Thirdly, examples show that the provided methods combining with the extreme value transmission theory can solve both the time-invariant problems like Example 2 and the timevariant problems like Example 3, which provide a general and feasible solution to these problems. Finally, the new proposed indices can provide more intuitive and compact index than ever, which establishes basement to have parametric reliability sensitivity analysis and importance analysis in further research. 


\section{Summary}

Based on the amount and type of available information, either probability theory or possibility theory can be used to describe different uncertainties. Whereas in most realistic applications, the uncertain variables cannot completely be assumed to be random variables or fuzzy variables in nature; random and fuzzy variables often exist simultaneously in engineering. Three reliability indices are proposed to intuitively and compactly represent the propagation of the uncertainty through the performance response function with a combination of random and fuzzy variables.

The PDEM-based solution is provided to avoid the tremendously computational cost in the process of computing these indices. The reasonability of the proposed indices and the efficiency of the presented PDEM-based methodology are demonstrated by the numerical and engineering examples through the comparison of the results of the PDEM-based method and those of Monte Carlo method.

In addition, the proposed reliability indices can provide intuitive and compact information to reflect the safety degree of the structure or mechanism containing mixed variables, which could provide a convenient approach to the reliability sensitivity analysis and importance analysis of parameters for such problems in engineering.

\section{Conflict of Interests}

The authors declare here that they have no conflict of interests in their paper.

\section{Acknowledgment}

The authors gratefully acknowledge the support of the Nature Science Foundation of China (NSFC 71401174).

\section{References}

[1] G. Apostolakis, "The concept of probability in safety assessments of technological systems," Science, vol. 250, no. 4986, pp. 1359-1364, 1990.

[2] J. C. Helton and W. L. Oberkampf, "Alternative representations of epistemic uncertainty," Reliability Engineering and System Safety, vol. 85, no. 1-3, pp. 1-10, 2004.

[3] E. Zio, "Reliability engineering: old problems and new challenges," Reliability Engineering and System Safety, vol. 94, no. 2, pp. 125-141, 2009.

[4] C. G. Bucher and U. Bourgund, "A fast and efficient response surface approach for structural reliability problems," Structural Safety, vol. 7, no. 1, pp. 57-66, 1990.

[5] R. V. Grandhi and L. P. Wang, "Higher-order failure probability calculation using nonlinear approximations," Computer Methods in Applied Mechanics and Engineering, vol. 168, no. 1-4, pp. 185-206, 1999.

[6] R. C. Penmetsa and R. V. Grandhi, "Structural system reliability quantification using multipoint function approximations," AIAA Journal, vol. 40, no. 12, pp. 2526-2531, 2002.

[7] V. Braibant, A. Oudshoorn, C. Boyer, and F. Delcroix, "Nondeterministic possibilistic approaches for structural analysis and optimal design," AIAA journal, vol. 37, no. 10, pp. 1298-1303, 1999.
[8] W. M. Dong and F. S. Wong, "Fuzzy weighted averages and implementation of the extension principle," Fuzzy Sets and Systems, vol. 21, no. 2, pp. 183-199, 1987.

[9] S. A. Smith, T. Krishnamurthy, and B. H. Mason, "Optimized vertex method and hybrid reliability," in Proceedings of the 43rd AIAA/ASME/ASCE/AHS/ASC Structures, Structural Dynamics and Materials Conference, Denver, Colo, USA, April 2002.

[10] R. C. Penmetsa and R. V. Grandhi, "Uncertainty propagation using possibility theory and function approximations," Mechanics Based Design of Structures and Machines, vol. 31, no. 2, pp. 257-279, 2003.

[11] B. Möller, W. Graf, and M. Beer, "Safety assessment of structures in view of fuzzy randomness," Computers and Structures, vol. 81, no. 15, pp. 1567-1582, 2003.

[12] T.-C. Lai, Y. N. Sotskov, N. Sotskova, and F. Werner, "Mean flow time minimization with given bounds of processing times," European Journal of Operational Research, vol. 159, no. 3, pp. 558-573, 2004

[13] N. M. Matsveichuk, Y. N. Sotskov, and F. Werner, "The dominance digraph as a solution to the two-machine flow-shop problem with interval processing times," Optimization, vol. 60, no. 12, pp. 1493-1517, 2011.

[14] P. R. Adduri and R. C. Penmetsa, "Confidence bounds on component reliability in the presence of mixed uncertain variables," International Journal of Mechanical Sciences, vol. 50, no. 3, pp. 481-489, 2008.

[15] P. R. Adduri and R. C. Penmetsa, "System reliability analysis for mixed uncertain variables," Structural Safety, vol. 31, no. 5, pp. 375-382, 2009.

[16] J. Li and J. B. Chen, "The probability density evolution analysis of stochastic dynamic system," Progress in Natural Science, vol. 16, no. 6, pp. 712-719, 2003.

[17] L. A. Zadeh, "Fuzzy sets," Information and Computation, vol. 8, no. 2, pp. 338-353, 1965.

[18] C. G. Bucher, "Adaptive sampling-an iterative fast Monte Carlo procedure," Structural Safety, vol. 5, no. 2, pp. 119-126, 1988.

[19] R. E. Melchers, "Importance sampling in structural systems," Structural Safety, vol. 6, no. 1, pp. 3-10, 1989.

[20] J. Nie and B. R. Ellingwood, "Directional methods for structural reliability analysis," Structural Safety, vol. 22, no. 3, pp. 233-249, 2000.

[21] J.-B. Chen and J. Li, "A note on the principle of preservation of probability and probability density evolution equation," Probabilistic Engineering Mechanics, vol. 24, no. 1, pp. 51-59, 2009.

[22] J. D. Anderson, Computational Fluid Dynamics, McGraw-Hill, 1995.

[23] L. G. Hua and Y. Wang, Applications of Number Theory to Numerical Analysis, Springer, Berlin, Germany, 1981.

[24] J. Li and J.-B. Chen, "The number theoretical method in response analysis of nonlinear stochastic structures," Computational Mechanics, vol. 39, no. 6, pp. 693-708, 2007.

[25] S. Song, Z. Lu, and H. Qiao, "Subset simulation for structural reliability sensitivity analysis," Reliability Engineering and System Safety, vol. 94, no. 2, pp. 658-665, 2009.

[26] Y. M. Zhang, X. Z. Huang, and X. D. He, "Reliability-based robust design for kinematic accuracy of the shaper mechanism under incomplete probability information," Journal of Mechanical Engineering, vol. 45, no. 4, pp. 105-110, 2009 (Chinese).

[27] J. Li, J.-B. Chen, and W.-L. Fan, "The equivalent extremevalue event and evaluation of the structural system reliability," Structural Safety, vol. 29, no. 2, pp. 112-131, 2007. 


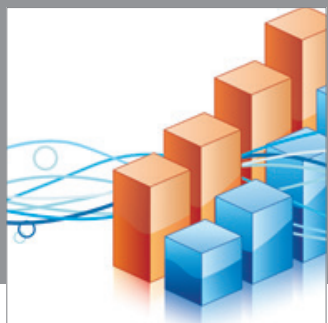

Advances in

Operations Research

mansans

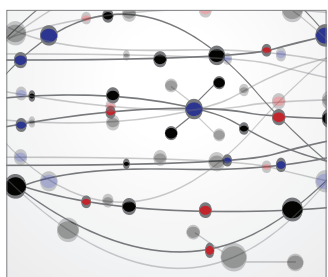

The Scientific World Journal
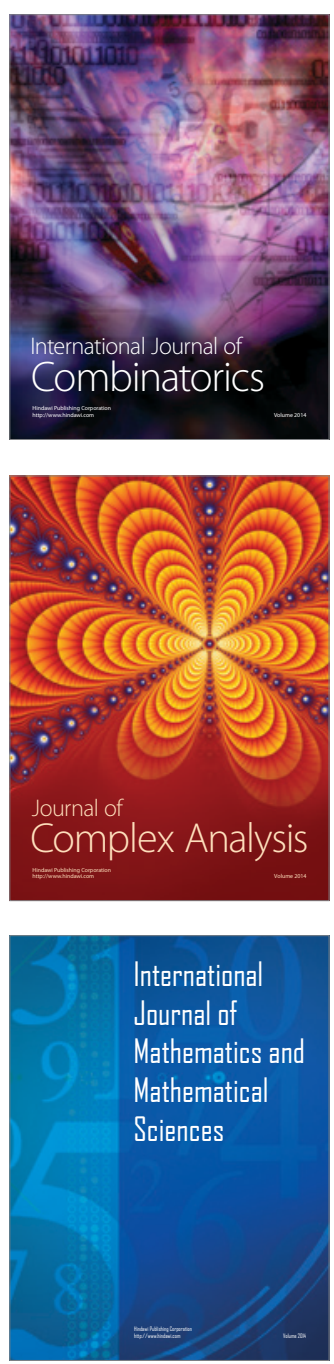
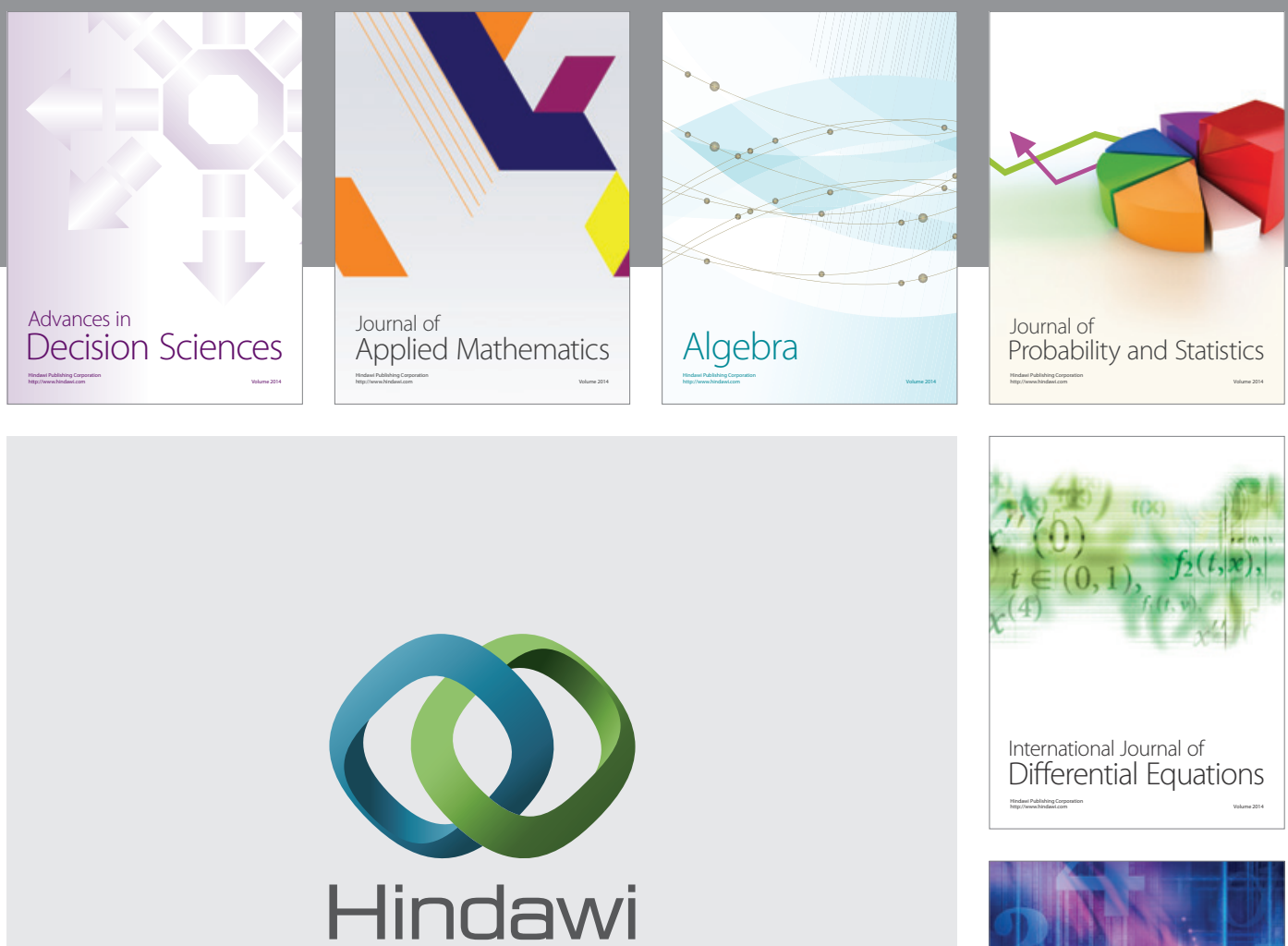

Submit your manuscripts at http://www.hindawi.com
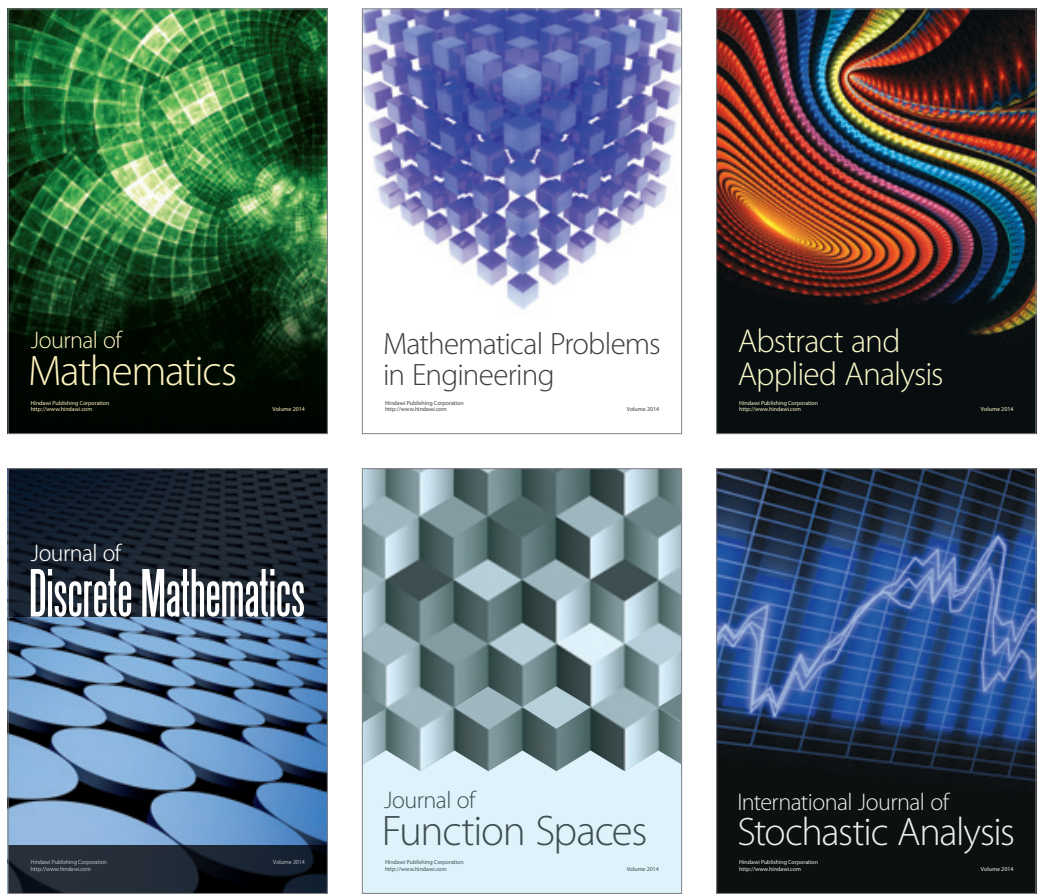

Journal of

Function Spaces

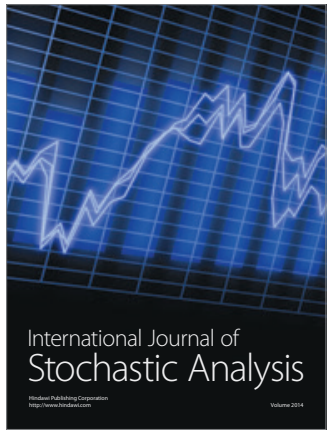

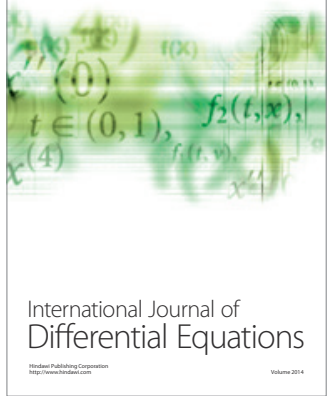
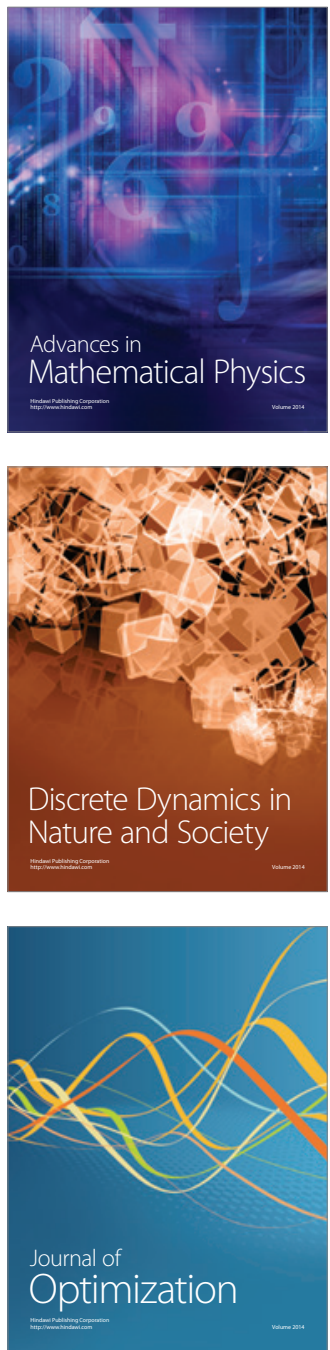